\title{
A Complete Review on Various Noises and Recent Developments in Denoising Filters
}

\author{
Taylor and Ba Jin
}

\begin{abstract}
In this paper, presented a complete tutorial review of recent developments in reduction of noise. Reduction of noise is a vital research topic in the field of image restoration. In accordance with several literatures, this paper attempts to formulate an overview of reduction of noise. Completely removing the noise from original image is still a tough task for researchers. There have been quite a lot of published schemes and each scheme has its own assumptions, merits and demerits. This paper presents a complete review of some significant work in the area of image denoising. Following a brief introduction, it portrays the theory associated with the categories of noises and subsequently this paper demonstrates some denoising schemes found in literature for noise reduction and a general idea of several algorithms and analysis is provided. The major emphasis here is on primary concepts and ideas rather than mathematical details.
\end{abstract}

Keywords--- Wavelet Transform, Gaussian Noise, Salt \& Pepper Noise, Speckle Noise, Guassian Filter, Wiener Filter.

\section{INTRODUCTION}

$\mathrm{I}_{\mathrm{a}}^{\mathrm{m}}$ MAGE processing is basically the use of computer algorithms to perform image processing on digital images. Digital image processing is a part of digital signal processing. Digital image processing has many significant advantages over analog image processing. Image processing allows a much wider range of algorithms to be applied to the input data and can avoid problems such as the build-up of noise and signal distortion during processing of images.

An image is a two dimensional function $\mathrm{f}(\mathrm{x}, \mathrm{y})$, where $\mathrm{x}$ and $y$ are plane coordinates, and the amplitude of $f$ at any pair of coordinates $(x, y)$ is called the gray level or intensity of the image at that point. Digital images consist of a finite number of elements where each element has a particular location and value. These elements are called picture elements, image elements and pixels. There are two types of images i.e. grayscale image and RGB image. Gray scale image has one channel and RGB image has three channels i.e. red, green and blue. Image noise is unwanted fluctuations.

Image filtering algorithms are applied on images to remove the different types of noise that are either present in the image during capturing or injected in to the image during transmission. Noise is introduced into images during acquisition, signal amplification and transmission. An important problem of image processing is to effectively

Taylor, The University of Texas at Austin, United States.

Ba Jin, University of Cambridge, United Kingdom.

DOI:10.9756/BIJPSIC.8332 remove noise from an image while keeping its features. Noise removal is a difficult task because images may be corrupted by different types of noise, such as additive, impulse or signal dependent noise. The solution depends upon the type of noise added to the image. However, linear techniques blur edges and fail for non-Gaussian and/or impulse noise.

Noise in an image is a very common problem. An image gets corrupted with different types of noise [2] during the processes of acquisition, transmission/ reception, and storage/ retrieval. Noise may be classified as substitutive noise (impulsive noise: e.g., salt\& pepper noise, random-valued impulse noise, etc.) and additive noise (e.g., additive white Gaussian noise)

\section{A. Image Noise}

The sources of noise in digital images arise during image acquisition and/or transmission with unavoidable shot noise of an ideal photon detector. The performance of imaging sensors are affected by a variety of factors during acquisition, such as

- Environmental conditions during the acquisition

- Light levels (low light conditions require high gain amplification).

- Sensor temperature (higher temp implies more amplification noise)

Depending on the specific noise source, there are different

\section{B. Additive and Multiplicative Noises}

Note that noise is undesired information that contaminates the image. Typical images are corrupted with noise modeled with either a Gaussian, uniform, or saltant pepper distribution. Another typical noise is a speckle noise, which is multiplicative in nature.

An additive noise follows the rule

$$
W(x, y)=s(x, y)+n(x, y),
$$

While the multiplicative noise satisfies

$$
W(x, y)=s(x, y) \times n(x, y),
$$

Where $s(x, y)$ is the original signal, $n(x, y)$ denotes the noise introduced into the signal to produce the corrupted image $w(x, y)$, and $(x, y)$ represents the pixel location

\section{TYPES OF NOISE}

\section{A. Gaussian Noise}

Gaussian noise is evenly distributed over the signal. This means that each pixel in the noisy image is the sum of the true pixel value and a random Gaussian distributed noise value. Gaussian noise is a noise that has its PDF equal to that of the normal distribution, which is also known as the Gaussian distribution. In other words, the values that the noise can take 
on are Gaussian-distributed. Gaussian noise is most commonly known as additive white Gaussian noise. Gaussian noise is properly defined as the noise with a Gaussian amplitude distribution. Labeling Gaussian noise as 'white' describes the correlation of the noise. It is necessary to use the term "white Gaussian noise" to be precise. A special case is white Gaussian noise [16, 17], in which the values at any pairs of times are statistically independent (and uncorrelated). In applications, Gaussian noise is most commonly used as additive white noise to yield additive white Gaussian noise.

\section{B. Salt-and-Pepper Noise}

Salt and pepper noise is a form of noise typically seen on images. It represents itself as randomly occurring white and black pixels. An effective noise reduction method for this type of noise involves the usage of a median filter or a contra harmonic mean filter. Salt and pepper noise creeps into images in situations where quick transients, such as faulty switching, take place [17]. Salt and pepper noise is an impulse type of noise, which is also referred to as intensity spikes. This is caused generally due to errors in data transmission.

\section{Speckle Noise}

Speckle noise is a multiplicative noise. This type of noise occurs in almost all coherent imaging systems such as laser, acoustics and SAR (Synthetic Aperture Radar) imagery. Speckle noise is caused by signals from elementary scatterers, the gravity-capillary ripples, and manifests as a pedestal image.

\section{Brownian Noise}

Brownian noise comes under the category of fractal or $1 / f$ noises. The mathematical model for $1 / f$ noise is fractional Brownian motion. Fractal Brownian motion is a nonstationary stochastic process that follows a normal distribution. Several different methods are used to eliminate speckle noise, based upon different mathematical models of the phenomenon.

\section{NOISE REDUCTION}

It is an important concern in image processing and computer vision. Images obtained from the real world are corrupted with noise. The image noise might decrease to some negligible levels under ideal conditions such that denoising is not necessary, but in general to recover the image the corrupting noise must be removed for practical purposes. Noise makes ambiguities in the underlying signal relative to its observed form by perturbations which are not related to the scene under study. The goal of denoising is to remove the noise and to retain the important signal features as much as possible. Most of the standard algorithms use to de-noise the noisy image and perform the individual filtering process. The result is that it generally reduces the noise level. But the image is either blurred or over smoothed due to losses like edges or lines.

Digital images play an important role both in day to-day applications, such as, satellite television, magnetic resonance imaging, computer tomography as well as in areas of research and technology such as geographical information systems and astronomy. In the diverse fields, mentioned above, scientists are faced with the problem of recovering original images from incomplete, indirect and noisy images. Generally, data sets collected by image sensors are contaminated by noise. Imperfect instruments, problems with the data acquisition process, and interfering natural phenomena can all degrade the data of interest. There are many different cases of distortions. One of the most prevalent cases is distortion due to additive white Gaussian noise which can be caused by poor image acquisition or by transferring the image data in noisy communication channels. Other types of noises include impulse and speckle noises. Furthermore, noise can be introduced by transmission errors and compression. Thus, denoising is often a necessary and the first step to be taken before the image data is analyzed. It is necessary to apply an efficient denoising technique to compensate for such data corruption.

Image denoising still remains a challenge for researchers because noise removal introduces artifacts and causes blurring of the images. Denoising of electronically distorted images is an old but also still a relevant industrial problem. In the past two decades, many methods for de-noising have been developed and reported in the literature. The process of removing noise from the original image is still a demanding problem for researchers. There have been several algorithms and each has its assumptions, merits, and demerits.

\section{IMAGE DE-NOISING FILTERS}

As discussed above there are two types of noise typically seen on images, Salt \& Peepers noise and Gaussian noise. To remove these noises various filters are used. Selection of appropriate filter for de-noising is depends upon the type of noise. So here in the following section firstly we are going to discuss the non linear filters which are widely used for image de-noising, Spatial domain filter for removing noise from digital images used for real time applications.

Linear filters, which consist of convolving the image with a constant matrix to obtain a linear combination of neighborhood values, have been widely used for noise elimination in the presence of additive noise. However they can produce a blurred and smoothed image with poor feature localization and incomplete noise suppression.

Gaussian filters are typical linear filters that have been widely used for image denoising. Gaussian filters assume that image signals have smooth spatial variations and pixels in a neighborhood have close values, so noise will be suppressed while signal will be preserved by averaging pixel values over a local neighborhood. The assumption of slow spatial signal variations works well in smooth regions; however it fails and undesirably blurs the signal where spatial variations are high such as edges. To overcome this shortcoming and prevent undesirable blurring in regions with high spatial signal variations, a number of filters in spatial and spatial-frequency domain are used. The most popular ones in spatial domain are anisotropic diffusion [1-3], bilateral filtering and its extensions [4-8]. Diffusion based methods iteratively solve partial differential equations and average the signal over spatial neighborhood whose extension is determined based on local 
signal variations. Gaussian filters are designed to give no overshoot to a step function input while minimizing the rise and fall time. This behavior of Gaussian filter causes minimum group delay. Mathematically, a Gaussian filter modifies the input signal by convolving with a Gaussian function. The Gaussian filter is usually used as a smoother. The output of the Gaussian filter at the moment is the mean of the input values [3].

Wiener Filter is used to reduce disturbance (noise) present in a signal by comparison with an estimation of the desired noiseless signal. The design of the Wiener filter is of different approach. The Wiener filtering is a linear estimation of the original image [4]. The approach is based on a stochastic framework. Wiener filters are characterized by the following:

1. Assumption: signal and (additive) noise are stationary linear with known spectral Characteristics

2. Requirement: the filter must be physically realizable/causal system.

3. Performance Criterion: minimum MMSE [5]

Nonlinear filters in spatial-frequency domain have also been used to preserve detail signal and suppress the noise. The most popular ones are wavelet based denoising techniques [912]. In wavelet based denoising methods, the noise is estimated and wavelet coefficients are thresholded to separate signal and noise. Various approaches to nonlinear waveletbased denoising have been introduced among them. Bayes shrink wavelet denoising is developed in the Bayesian framework and has been widely used for image denoising [1011]. An adaptive technique is used to tune the extensions of range and domain filters. The distance of the local histogram from the estimated noise histogram is measured using earth mover's distance. The measured distance at each spatial location is then used for adaptive tuning of bilateral filter. This method effectively removes the noise while preserves the signal character.

\section{A. Fourth Order Statistics Filter}

Usually, sliding window technique [4] is employed to perform pixel-by-pixel operation in a filtering algorithm. The local statistics obtained from the neighbor-hood of the centre pixel give a lot of information about its expected value. If the neighbor-hood data are ordered (sorted), then ordered statistical information is obtained. If this order statistics vector is applied to a finite impulse response (FIR) filter, then the overall scheme becomes an order statistics (OS) filter.

\section{B. Mean and Median Filters}

The moving average or mean filter (MF) is a simple linear filter [5]. All the input data are summed together and then the sum is divided with the number of data. It is very simple to implement in hardware and software. The computational complexity is very low. It works fine for low power AWGN [12]. As the noise power increases, its filtering performance degrades. If the noise power is high, then a larger window should be employed for spatial sampling to have better local statistical information. As the window size increases, MF produces a reasonably high blurring effect and thus thin edges and fine details in an image are lost. The median (MED) filter $[10,11]$. On the other hand, is a nonlinear filter. Usually, such a computation is not required in most of image processing applications as the window length is normally an odd number. Thus, the MED operation can be completed in a very short time. That is, a MED filter may be used for online and real time applications to suppress noise. If an image is corrupted with a very low variance AWGN, then this filter can perform a good filtering operation.

A mean filter acts on an image by smoothing it; that is, it reduces the intensity variation between adjacent pixels. The mean filter is nothing but a simple sliding window spatial filter that replaces the center value in the window with the average of all the neighboring pixel values including itself. By doing this, it replaces pixels that are unrepresentative of their surroundings. It is implemented with a convolution mask, which provides a result that is a weighted sum of the values of a pixel and its neighbors. It is also called a linear filter. The mask or kernel is a square. Often a $3 \times 3$ square kernel is used. If the coefficients of the mask sum up to one, then the average brightness of the image is not changed. If the coefficients sum to zero, the average brightness is lost, and it returns a dark image. The mean or average filter works on the shift-multiplysum. Mean filter, or average filter is windowed filter of linear class, that smoothes signal (image). The filter works as lowpass one. The basic idea behind filter is for any element of the signal (image) take an average across its neighborhood. To understand how that is made in practice, let us start with window idea. The Average (mean) filter smooths image data, thus eliminating noise [6]. This filter performs spatial filtering on each individual pixel in an image using the grey level values in a square or rectangular window surrounding each pixel [5]. For example: a1 a2 a3 a4 a5 a6 3x3 filter window a7 a8 a9 The average filter computes the sum of all pixels in the filter window and then divides the sum by the number of pixels in the filter window: Filtered pixel $=(a 1+a 2+a 3+a 4$ $\ldots+$ a9) / 9. The Mean Filter is a linear filter which uses a mask over each pixel in the signal. Each of the components of the pixels which fall under the mask are averaged together to form a single pixel. This filter is also called as average filter. The Mean Filter is poor in edge preserving. Generally linear filters are used for noise suppression. It gives minimum PSNR when compared to non linear filters.

A median filter belongs to the class of nonlinear filters unlike the mean filter. The median filter also follows the moving window principle similar to the mean filter. A $3 \times 3$, $5 \times 5$, or $7 \times 7$ kernel of pixels is scanned over pixel matrix of the entire image. The median of the pixel values in the window is computed, and the center pixel of the window is replaced with the computed median. Median filtering is done by, first sorting all the pixel values from the surrounding neighborhood into numerical order and then replacing the pixel being considered with the middle pixel value. Note that the median value must be written to a separate array or buffer so that the results are not corrupted as the process is performed. The Median Filter is performed by taking the magnitude of all of the vectors within a mask and sorted according to the magnitudes. The pixel with the median magnitude is then used to replace the pixel studied. The Simple Median Filter has an advantage over the Mean filter since median of the data is taken instead of the mean of an 
image. A major advantage of the median filter over linear filters is that the median filter can eliminate the effect of input noise values with extremely large magnitudes. (In contrast, linear filters are sensitive to this type of noise - that is, the output may be degraded severely by even by a small fraction of anomalous noise values).

\section{Component Median Filter (CMF)}

CMF is defined in (3), relies on the statistical median concept. In the Simple Median Filter, each point in the signal is converted to a single magnitude. In the Component Median Filter each scalar component is treated independently. A filter mask is placed over a point in the signal. When noise affects a point in a gray scale image, the result is called "salt and pepper" noise.

\section{- Advantage}

The Component Median Filter is more accurate than the Simple Median Filter.

\section{The Vector Median Filter (VMF)}

In the VMF, a filter mask is placed over a single point. The point with the minimum sum of vector differences is used to represent the point in the signal studied.

\section{- Advantage}

The advantage of this method is that filter outputs are close to each other and can be manipulated by 2Dtechniques.

\section{E. Spatial Median Filter}

The SMF is a new noise removal filter. The SMF and the VMF follow a similar algorithm and it will be shown that they produce comparable results. The SMF is a uniform smoothing algorithm with the purpose of removing noise and fine points of image data while maintaining edges around larger shapes

\section{- Advantage}

The advantage of replacing every point achieves a uniform smoothing across the image. A good smoothing filter should simplify the image while retaining most of the original image shape and retain the edges.

\section{F. Modified Spatial Median Filter for Gaussian Noise}

The SMF is similar to the VMF in that in both filters, the vectors are ranked by some criteria and the top ranking point is used to replace the center point. No consideration is made to determine if that center point is original data or not. The unfortunate drawback of these filters is the smoothing that occurs uniformly across the image. Across areas where there is no noise, uncorrupted data is removed unnecessarily.

\section{- Advantage}

Modified spatial Median filter median filter is the most suitable filter for denoising the images for different type of noise.

\section{G. Kuan Filter}

The Kuan filter is used primarily to filter speckled radar data. It is designed to smooth out noise while retaining edges or shape features in the image. Kuan filter [9] also smoothes the image without removing edges or sharp features in the images. It is only applicable for radar intensity image. Kuan filter first transforms the multiplicative noise model into a signal-dependent additive noise model. Then the minimum mean square error criterion is applied to the model. The resulting filter has the same form as the Lee filter but with a different weighting function. Because Kuan filter made no approximation to the original model, it can be considered to be superior to the Lee filter in this paper, various spatial-domain filters for suppression of salt and peppers noise \& Zero-Mean Gaussian White Noise (AWGN), available in literature, are studied and their performances are analyzed.

\section{H. LMS Adaptive Filter}

An adaptive filter does a better job of denoising images compared to the averaging filter. The fundamental difference between the mean filter and the adaptive filter lies in the fact that the weight matrix varies after each iteration in the adaptive filter while it remains constant throughout the iterations in the mean filter. Adaptive filters are capable of denoising non-stationary images, that is, images that have abrupt changes in intensity. Such filters are known for their ability in automatically tracking an unknown circumstance or when a signal is variable with little a priori knowledge about the signal to be processed. In general, an adaptive filter iteratively adjusts its parameters during scanning the image to match the image generating mechanism. This mechanism is more significant in practical images, which tend to be nonstationary. Compared to other adaptive filters, the Least Mean Square (LMS) adaptive filter is known for its simplicity in computation and implementation. The basic model is a linear combination of a stationary low-pass image and a nonstationary high-pass component through a weighting function. Thus, the function provides a compromise between resolution of genuine features and suppression of noise.

\section{Wavelet Domain Filtering}

Wavelets are mathematical functions that cut up data into different frequency components, and then study each component with a resolution matched to its scale. They have advantages over traditional Fourier methods in analyzing physical situations where the signal contains discontinuities and sharp spikes [20]. Wavelets were developed independently in the fields of mathematics, quantum physics, electrical engineering, and seismic geology. Interchanges between these fields during the last ten years have led to many new wavelet applications such as image compression, turbulence, human vision, radar, and earthquake prediction [12][18].

Wavelets provide some advantages over Fourier transforms. For example, they do a good job in approximating signals with sharp spikes or signals having discontinuities. Wavelets can also model speech, music, video and nonstationary stochastic signals. Wavelets can be used in applications such as image compression, turbulence, human vision, radar, earthquake prediction, etc. The term "wavelets" is used to refer to a set of orthonormal basis functions generated by dilation and translation of scaling function $\varphi$ and a mother wavelet Mallat's algorithm is a computationally efficient method of implementing the wavelet transform. It calculates DWT wavelet coefficients for a finite set of input data, which is a power of 2. Denoising of images using VisuShrink, Sure Shrink and Bayes Shrink using Matlab 6.1 is 
discussed in this chapter. All these methods are based on the application of wavelet transforms.

Noise reduction is used to remove the noise without losing much detail contained in an image [2]. To achieve this goal, we use the mathematical function known as the wavelet transform to localize an image into different frequency components or useful sub-bands and effectively reduce the noise in the sub-bands into different frequency components or useful sub-bands and effectively reduces the noise in the subbands.

\section{Discrete Wavelet Transform}

Wavelets are functions generated from one single function $\Psi$ by dilations and translations. The basic idea of the wavelet transform is to represent any arbitrary function as a superposition of wavelets. Any such superposition decomposes the given function into different scale levels ere each level is further decomposed with a resolution adapted to that level.

Wavelet Analysis, a new form of signal analysis is far more efficient than Fourier analysis wherever a signal is dominated by transient behavior or discontinuities. Several investigations have been made into additive noise suppression in signals and images using wavelet transforms. Much of the early work on wavelet noise removal based on thresholding the Discrete Wavelet Transform (DWT) coefficients of an image and then reconstructing it, was done by Donoho and Johnstone [9]. It has been found that wavelet based denoising is effective in that although noise is suppressed, edge features are retained without much damage.

A wavelet transform is the representation of a function by wavelets. The wavelets are scaled and translated copies of a mother wavelet. Wavelet analysis represents the next logical step: a windowing technique with variable-sized regions. Wavelet analysis allows the use of long time intervals where we want more precise low-frequency information, and shorter regions where we want high frequency information. Wavelet transforms are classified into discrete wavelet transforms (DWTs) and continuous wavelet transforms (CWTs). Both DWT and CWT are continuous-time (analog) transforms. They can be used to represent continuous-time (analog) signals. CWTs operate over every possible scale and translation whereas DWTs use a specific subset of scale and translation values or representation grid

\section{Multifractal Image Denoising}

The most recent techniques in image denoising known as multifractal analysis. Multifractal structures are generated by the multiplicative cascade of random processes, while additive processes generally produce simple fractals or mono fractals. Functions that are everywhere continuous but nowhere differentiable are called fractals. They are objects of a complex structure, which exhibit the scaling property, that is, they exhibit the same properties at different scales. A fractal describes the local singularity and is usually measured using the Hurst parameter. The fractal dimension is the basic notion for describing structures that have scaling symmetry and is closely related to Holder regularity (see Section 5.2). Fractal dimension is a non-integer value. Multifractal analysis gives a compact representation of the spectral decomposition of a signal into parts of equal strength of regularity. This property makes multifractals very useful in image denoising. Other applications of multifractals are in the fields of turbulence, rainfall, dynamical systems and in earthquake modeling.

\section{Multifractal Analysis}

A lot of research is going on in the use of mutlifractal analysis in image denoising. Denoising is done based on factors such as spectrum shift value and Holder exponent shift of the input signal. Two methods for image denoising using multifractal analysis are considered here. They are multifractal regularization [Ve01] and multifractal pumping

\section{Multifractal Regularization}

Multifractal regularization is a process by which the Holder regularity of the input image is increased by the use of a wavelet-based approach. As mentioned above, according to the functional analysis point of view, no assumptions about the noise structure are made. A regularized version of the observed data is obtained that fulfills some constraints.

\section{Multifractal Pumping}

Multifractal pumping is a procedure by which the Holder exponent of a received signal is increased so that the regularity of the signal is improved and the signal is closet the desired signal. In this method, initially, a wavelet transform is applied and the image is decomposed into its wavelet coefficients. The wavelet coefficients obtained from the wavelet transform at scale $j$ are multiplied by $2-\delta$ this chapter considered one of the latest techniques in denoising of images, i.e.,multifractal analysis. This method is very helpful for the removal of noise from an image that has a complex and irregular nature. This method finds applications in denoising of Synthetic Aperture Radar (SAR) images. It is also observed that multifractal pumping is more effective than multifractal regularization.

\section{J. Edge-Preserving Smoothing Filter}

When the spatial smoothness forces the illumination image where passing from a strongly illuminated region to a dark region, the illumination remain high in the dark region near the edge and smoothly descend to grasp the illumination within the dark region. Thus, when removed, the dark regions near such edges remain dark, resulting in the HALO effects. Such effects can also be obtained in the bright areas near such illumination edges. Also, the contrast stretching causes a magnification of the noise in dark regions, which becomes evident especially in low-quality images [7]. The edge preserving smoothing filter is generally forced to be used to solve the HALO and noise

\section{Bilateral Filter}

The idea of bilateral filtering has been adopted for the many applications not only in the area of image de-noising. Bilateral filtering was introduced by Tomasi and Manduchi [4] to smooth noisy images while preserve edges using neighboring pixels. Bilateral filtering is a local, nonlinear, and noniterative technique which considers both gray level (color) similarities and geometric closeness of the neighboring pixels. In a traditional domain filter, weight of the pixels decays by distance from the center of the filter. Low pass filters assume 
that spatial variations is slow over the image, so by weighted averaging of pixel values in a neighborhood, noise will be averaged away while the signal will be preserved. However, this assumption fails at edges where the spatial variations are not smooth and application of the low pass filter blurs the edges. Bilateral filter overcomes this by filtering the image in both range and domain. Bilateral filtering also known as range and domain filtering is a non-linear filter which performs weighted averaging in both range and domain. Pixels in a neighborhood are considered close either based on their spatial location (domain), or based on their pixel values (range). Therefore bilateral filter averages pixel values based on weights that decay by both distance and pixel dissimilarity. There are several extensions to improve bilateral filtering [58]. In [6], a training-based bilateral filtering is a general degradation model is considered for degraded images. Then A restoration algorithm is developed to restore the degraded images with unknown degradation process. Therefore, the success of restoration process depends on the general definition of degradation model. In [7, 8], different methods to speed up the bilateral filtering have been used. Bilateral filter combines range and domain filtering where the range filter averages the signal values in a neighborhood by assigning the weights based on the similarity of the neighboring pixels and the central pixel. Bilateral filtering overcomes the shortcomings of linear domain filtering by combining the linear domain filter with a nonlinear range filter. As a result bilateral filter preserves signal details such as edges while suppresses noise.

\section{Sigma Filter}

Lee [25] introduced a spatial filter that is based on the Sigma probability of the Gaussian distribution. The sigma filter identifies impulse noise for noisy gray scale images by utilizing the standard deviation measure,

\section{Mean Shift Filter}

The mean shift filter for the feature selection, object tracking [27], and noise removal [28] is a nonparametric feature space clustering technique that does not require prior knowledge of the number of clusters, and does not constrain the shape of the clusters

\section{K. Adaptive Range and Domain Filtering}

In this, to improve the performance of bilateral filtering, spatial noise level is locally estimated to determine the filter parameters. To estimate the local spatial noise level, the image noise histogram is estimated and compared with the local signal. To compare two probability density functions (PDFs), a number of nonparametric models have been used including minimizing the comparison function between two PDFs. The earth mover's distance (EMD) or the Wasserstein distance is a mathematical measure to compare distributions (histograms).

The original bilateral filter, and a popular wavelet denoising method so called Bayes shrink wavelet denoising were applied to the corrupted test images. In this paper an adaptive range and domain filtering method based on local histograms was introduced. The noise histogram is estimated and the extensions of range and domain filters are tuned at each spatial location by measuring the distance between the pixel's and noise histograms using earth mover's distance.

There are two basic approaches to image denoising, spatial filtering methods and transform domain filtering methods. Spatial filters employ a low pass filtering on groups of pixels with the assumption that the noise occupies the higher region of frequency spectrum. Spatial Low-pass filters will not only smooth away noise but also blur edges in signals and images while the high-pass filters can make edges even sharper and improve the spatial resolution but will also amplify the noisy background [8]. The conventional Fast Fourier Transform (FFT) based image denoising method is essentially a low pass filtering technique in which edge is not as sharp in the reconstruction as it was in the original. The edge information is spread across frequencies because of the FFT basis functions, which are not being localized in time or space. Hence low pass-filtering results in the smearing of the edges. But, the localized nature of the wavelet transforms both in time and space results in denoising with edge preservation.

Adaptive speckle filtering is better at preserving edges and detail in high-texture areas (such as forests or urban areas) [8] [22]. Non-adaptive filtering is simpler to implement, and requires less computational power. There are two forms of non-adaptive speckle filtering: one based on the mean and other based upon the median (within a given rectangular area of pixels in the image). The latter is better at preserving edges while eliminating noise spikes, than the former.

\section{PARAMETRIC DESCRIPTION}

A quantitative measure of comparison is provided by the parameters like Peak signal to noise ratio, Root mean square error and Correlation of the image.

\section{A. Algorithm for Peak Signal to Noise ratio (PSNR)}

- $\quad$ Step1: Difference of noisy image and noiseless image is calculated using imsubract Command.

- Step2: Size of the matrix obtains in step 1 is calculated.

- Image De-Noising using Wavelet Transform and Various Filters 17 www.ijorcs.org

- $\quad$ Step3: Each of the pixels in the matrix obtained in step is squared.

- Step4: Sum of all the pixels in the matrix obtained in Step3 is calculated.

- Step5: (MSE) is obtained by taking the ratio of value obtained in step 4 to the value obtained in the Step2

- Step6: (RMSE) is calculated by taking square root to the value obtained in Step5.

- $\quad$ Step7: Dividing 255 with RMSE, taking $10 g$ base 10 and multiplying with 20 gives the value of PSNR.

\section{B. Algorithm for Correlation of Coefficient (Coc)}

- Step1: Mean of the noiseless image and noisy image are calculated.

- Step2: Mean of the noiseless image is subtracted from each of the pixel in the noiseless image resulting in a matrix. 
- Step3: Similarly the mean of noisy image is subtracted from each of the pixels in the noise image resulting in a matrix.

- Step4: Values obtained in Step2 and Step3 are multiplied.

- Step5: Sum of all the elements in the matrix obtained in Step4 is calculated.

- Step6: Square of all the elements of the matrix obtained in Step2 is calculated and sum of this squared matrix is determined.

- Step7: Similarly square of all the elements of the matrix obtained in Step3 is calculated and sum of the elements of this squared matrix is also determined.

- Step8: Values obtained in Step6 and Step7 are multiplied and its square root is taken.

- Step9: Ratio of the value obtained in Step5 to the value obtained in Step8 is calculated.

\section{Algorithm for Root Mean Square Error (RMSE)}

- Step1: Difference of noisy image and noiseless image is calculated using imsubractcommand.

- Step2: Size of the matrix obtains in Step1 is calculated.

- Step3: Each of the pixels in the matrix obtained in step is squared.

- Step4: Sum of all the pixels in the matrix obtained in step 3 is calculated.

- Step5: (MSE) is obtained by taking the ratio of value obtained in Step4 to the value obtained in the step 2.

- Step6: (RMSE) is calculated by taking square root to the value obtained in Step5.

\section{Algorithm for Filter Selection}

- Step1: Noiseless image are given as input.

- Step2: Noisy image are then given as input.

- Step3: Noisy image is filtered by all the filters i.e. Gaussian, average, wiener and wavelet filter with respect to the noiseless image.

- Step4: The statistical parameters are calculated for the filtered image obtained from filtering

- Step5: Finally we get sets of statistical parameters.

\section{E. Local Histograms}

They are computed to tune the range and domain extensions of bilateral filter. Noise histogram is estimated to measure the noise level at each pixel in the noisy image.

\section{CONCLUSION}

Most of the methods described above are used for removal of noise for still images and are computationally complex for low bit rate applications like real time mobile image for portable devices and image conferencing on Internet over low bandwidth lines. Performance of denoising algorithms is measured using quantitative performance measures such as peak signal-to-noise ratio (PSNR), signal-to-noise ratio (SNR) as well as in terms of visual quality of the images. Many of the current techniques assume the noise model to be Gaussian. In reality, this assumption may not always hold true due to the varied nature and sources of noise. An ideal denoising procedure requires a priori knowledge of the noise, whereas a practical procedure may not have the required information about the variance of the noise or the noise model. Thus, most of the algorithms assume known variance of the noise and the noise model to compare the performance with different algorithms. Gaussian Noise with different variance values is added in the natural images to test the performance of the algorithm. Not all researchers use high value of variance to test the performance of the algorithm when the noise is comparable to the signal strength.

\section{REFERENCES}

[1] M. Kazubek, "Wavelet domain image de-noising by thresholding and Wiener filtering", Signal Processing Letters, Vol. 10, No. 11, 2003.

[2] D.L. Donoho, "Wavelet shrinkage and WVD: A 10-minute tour", International Conference on Wavelets and Applications, 1992.

[3] S. Zhong and V. Cherkassky, "Image denoising using wavelet thresholding and model selection”, International Conference on Image Processing, Vol. 3, Pp. 262-265, 2000.

[4] C. Boncelet, "Image Noise Models", Alan C.Bovik. Handbook of Image and Video Processing, 2005.

[5] S. Kent, O.N. Oçan and T. Ensari, "Speckle Reduction of Synthetic Aperture Radar Images Using Wavelet Filtering”, Proceedings, 5th European Conference on Synthetic Aperture Radar on ITG, VDE, FGAN, DLR, EADS, astrium, 2004.

[6] J.C. Church, Y. Chen and S.V. Rice, "A Spatial Median Filter for Noise Removal in Digital Images”, Southeastcon, Pp. 618- 623, 2008.

[7] H. Guo, J. E. Odegard, M. Lang, R.A. Gopinath, I.W. Selesnick and C.S. Burrus, "Wavelet based speckle reduction with application to SAR based ATD/R”, First Int'l Conf. on Image Processing, Vol. 1, Pp. 75-79, 1994.

[8] R.D. Nowak, "Wavelet Based Rician Noise Removal”, IEEE Transactions on Image Processing, Vol. 8, No. 10, 1999.

[9] S.G. Mallat and W.L. Hwang, "Singularity detection and processing with wavelets”, IEEE Trans. Inform. Theory, Vol. 38, Pp. 617-643, 1992.

[10] D.L. Donoho, “De-noising by soft-thresholding”, IEEE Trans. Information Theory, Vol. 41, No. 3, Pp. 613-627, 1995.

[11] I.K. Fodor and C. Kamath, "Denoising through wavlet shrinkage: An empirical study", Center for applied science computing Lawrence Livermore National Laboratory, 2001.

[12] R. Coifman and D. Donoho, "Translation invariant de-noising", Lecture Notes in Statistics: Wavelets and Statistics, 1995.

[13] R. Yang, L. Yin, M. Gabbouj, J. Astola and Y. Neuvo, "Optimal weighted median filters understructural constraints”, IEEE Trans. Signal Processing, Vol. 43, Pp. 591-604, 1995.

[14] R.C. Hardie and K.E. Barner, "Rank conditioned rank selection filters for signal restoration”, IEEE Trans.Image Processing, Vol. 3, Pp.192-206, 1994.

[15] A. Ben Hamza, P. Luque, J. Martinez and R.Roman, "Removing noise and preserving details with relaxed median filters", J. Math. Imag. Vision, Vol. 11, No. 2, Pp. 161-177, 1999.

[16] A.K.Jain, "Fundamentals of digital image processing", Prentice-Hall, 1989

[17] D.L. Donoho and I.M. Johnstone,"Ideal spatial adaption via wavelet shrinkage”, Biometrika, Vol. 81, Pp. 425-455, 1994.

[18] D.L. Donoho and I.M. Johnstone.," Adapting to unknown smoothness via wavelet shrinkage”, Journal of the American Statistical Association, Vol. 90, No. 432, Pp. 1200-1224, 1995.

[19] V. Strela, "Denoising via block Wiener filtering in wavelet domain”, 3rd European Congress of Mathematics, Barcelona, 2000.

[20] H. Choi and R.G. Baraniuk, "Analysis of wavelet domain Wiener filters”, IEEE Int. Symp. Time-Frequency and Time-Scale Analysis, (Pittsburgh), 1998.

[21] H. Zhang, A. Nosratinia and R.O. Wells, "Image denoising via waveletdomain spatially adaptive FIR Wiener filtering”, IEEE Proc. Int. Conf. Acoust., Speech, Signal Processing, 2000.

[22] E.P. Simoncelli and E.H. Adelson, "Noise removal via Bayesian wavelet coring”, Third Int'l Conf on Image Proc, 1996. 
[23] H.A. Chipman, E.D. Kolaczyk and R.E.McCulloch, “Adaptive Bayesian wavelet shrinkage”, J.Amer. Stat. Assoc., Vol. 92, No. 440, Pp 1413-1421, 1997.

[24] M. Jansen, "Wavelet thresholding and noise reduction", Ph.D. Thesis, 2000.

[25] M. Lang, H. Guo, J.E. Odegard and C.S. Burrus, "Nonlinear processing of a shift invariant DWT for noise reduction", SPIE, Mathematical Imaging: Wavelet Applications for Dual Use, 1995.

[26] I. Cohen, S. Raz and D. Malah, "Translationinvariant denoising using the minimum description length criterion”, Signal Processing, Vol. 75, No. 3, Pp. 201-223, 1999.

[27] T.D. Bui and G.Y. Chen, "Translation-invariant denoising using multiwavelets", IEEE Transactions on Signal Processing, Vol. 46, No. 12, Pp. 3414-3420, 1998.

[28] R.G. Baraniuk, "Optimal tree approximation with wavelets”, Proc. SPIE Tech. Conf.Wavelet Applications Signal Processing VII, 1999.

[29] J. Lu, J. B.Weaver, D.M. Healy and Y. Xu, "Noise reduction with multiscale edge representation and perceptual criteria”, Proc. IEEE-SP Int. Symp. Time-Frequency and Time-Scale Analysis, 1992.

[30] D.L. Donoho, "CART and best-ortho-basis: A connection", Ann. Statist., Pp. 1870-1911, 1997. 